gentumsverhältnisse zu klären, musste das LG München I auch den Inhalt der vertraglichen Vereinbarungen ermitteln. Da es einen Schadensersatzanspruch aus Gefälligkeitsverwahrung (richtigerweise: aus Leihvertrag) bejaht hat, hätte es sich die teilweise falschen Ausführungen zum deliktischen Schadensersatzanspruch sparen können. Ohne praktische Relevanz für die hier behandelten Fälle zu haben, bergen die gesetzlichen Ansprüche juristischen Sprengstoff:

Zum einen ist das Verhältnis zwischen der vertraglichen Abwicklung und den Ansprüchen aus dem Eigentümer-Besitzer-Verhältnis (EBV, vgl. § 987 ff. BGB) bei weitem nicht geklärt. ${ }^{38}$ Zum anderen ergibt sich beim deliktischen Schadensersatz (§§ 823, 831 BGB) folgendes Problem: Die Stadt München haftet als juristische Person des öffentlichen Rechts im Rahmen deliktischer Ansprüche nicht für das individuelle

38 Auch der BGH urteilt uneinheitlich, das Verhältnis ist entgegen dem LG München I also nicht „ganz h.M.“; vgl. Palandt-Bassenge BGB, Vorb v $\S 987$ Rn. $10 \mathrm{mwN}$.
Verschulden ihrer Angestellten, jedenfalls nicht so unproblematisch wie es das LG angenommen hat. Die Stadt haftet nur für die Handlungen seiner Organe und leitenden Angestellten, nicht aber für das Handeln einfacher Angestellter (§§ 89, 31 BGB). ${ }^{39}$ Erstere müssen eigene Pflichten verletzen, insbesondere die Angestellten nicht sorgfältig ausgesucht, instruiert, organisiert oder überwacht haben (sog. Organisationsverschulden). Deren lückenlose Überwachung wird aber nicht verlangt. ${ }^{40}$ Ob die Stadt München Sorgfaltspflichten verletzt hat, hätte in einer ausführlichen Beweiserhebung festgestellt werden müssen und konnte nicht so einfach angenommen werden. Das allerdings wäre völlig unnötig gewesen, nachdem der vertragliche Anspruch bejaht wurde, weil die gesetzlichen Ansprüche dem Ausstellungsveranstalter hier keine anderen oder weitergehenden Pflichten auferlegen als ein Vertrag, die Verjährungsfristen nicht günstiger sind und auch kein weitergehender Schaden ersetzt wird.

39 Vgl. MünchKomm-G.Wagner BGB, § 823 Rn. 368.

40 Vgl. MünchKomm-G.Wagner BGB, § 823 Rn. 370 mwN.

\title{
Rechtsprechung
}

\section{Rechtsbeziehungen bei Auftragswerk für temporäre Kunstausstellung}

Landgericht München I, Urteil vom 04. April 2008 - 230 6414/07

\section{Das Eigentum an einem Auftragswerk für eine Kunstausstellung geht bei fehlender Vereinbarung nicht notwendig vom Künstler auf den Ausstellungsorganisator (Besteller) über.}

2. Der für eine Ersitzung ( $\$ 937$ BGB) eines Kunstwerkes durch ein städtisches Museum notwendige Eigenbesitz setzt voraus, dass das Kunstwerk inventarisiert wurde.

\section{Die Nichtinventarisierung eines Kunstwerkes durch ein städtisches Museum kann eine Pflichtverletzung} darstellen. (Leitsätze der Redaktion)

\section{Tatbestand}

- Ein Künstler begehrt nach 15 Jahren von einem städtischen Museum Schadensersatz für ein vermeintlich ihm gehörendes unauffindbares Kunstwerk.

Der Kläger ist ein renommierter Künstler, der als deutscher Staatsbürger in den USA lebt. Im Herbst 1991 (!) hat er durch das Kunstwerk „XX“ Aufsehen erregt, das Kunstwerk war am [...] aufgestellt. Da das Interesse an diesem Kunstwerk sehr hoch war, sollte der Künstler auch für die städtische Galerie Lenbachhaus in dem Projekt "ArgusAuge" mit einem weiteren Kunstwerk tätig werden. Es sollten dazu neue Fahnen geschaffen werden. Es kam im Folgenden zu einer Vereinbarung, genannt „Engagement", zwischen dem Kläger und der Stadt München, wonach er 5.000,00 DM Honorar für die Erstellung eines neuen Kunstwerkes und ein Budget von 20.000,00 DM zur Verfügung gestellt bekam.
In dieser Vereinbarung heißt es in $\S 1$ :

\section{"Vertragsgegenstand}

Leistung (Beschreibung) Vorbereitung einer Arbeit für das ISARProjekt '91 [...]"

Sowie bei den Allgemeinen Vertragsbedingungen unter 6.:

"Soweit in der Vereinbarung keine anderen Regelungen getroffen sind, gelten die Bestimmungen des $B G B$, insbesondere die $\S \S$ 632 ff. BGB."

Weitere ausdrückliche Vereinbarungen zum Eigentum oder dem Urheberrecht an diesem neu zu erstellenden Kunstwerk wurden weder schriftlich noch mündlich in irgendeiner Form fixiert. 
Das Kunstwerk "Die Fahnen hoch" hatte vor dem Hintergrund des ersten Golfkrieges/ Irakkrieges im Jahr 1991 unter zahlreichen anderen Firmen auch die Firma R. AG als vermeintlichen deutschen Unterstützer des Regimes im Irak genannt. Deshalb betrieb die R. AG noch während des Laufes der Ausstellung ein einstweiliges Verfügungsverfahren gegen die Landeshauptstadt München; dieses Verfahren im einstweiligen Rechtsschutz endete damals mit einem Vergleich der Parteien, der die Landeshauptstadt München verpflichtete, die genannten Fahnen nicht mehr zu zeigen. [...] Die Beklagte nahm daher die Fahnen aus der Ausstellung. Die Fahnen wurden anschließend nicht im Inventar des Museums erfasst, d. h. weder für das Museum selbst, noch für den Kläger. Aus dem Januar 1992 datiert dann noch eine Anfrage des Klägers nach dem Verbleib der Fahnen, ein Versand der Fahnen an den Kläger fand jedoch nicht statt. Was mit dem Kunstwerk geschah, konnte nicht nachvollzogen werden.

Anlässlich seines 70. Geburtstags im Jahre 2006 wandte sich der Kläger wieder an die Landeshauptstadt München, respektive die Galerie Lenbachhaus, und forderte die Herausgabe der Fahnen. Diese waren inzwischen bei der städtischen Galerie am Lenbachhaus nicht mehr auffindbar. Eine Herausgabe erfolgte nicht. Stattdessen ließ der Kläger zunächst auf eigene Rechnung neue Fahnen herstellen, was ihn in den USA 14.386,78 US-Dollar kostete.

Der Kläger verlangt nun Schadensersatz für die Neuerstellung. Er beruft sich zunächst auf künstlerische Übung, es sei zwischen Künstlern und den jeweiligen Museen, die Ausstellungen veranstalten, üblich, dass das Kunstwerk nach Beendigung der Ausstellung seitens des Museums in Verwahrung genommen wird. Üblicherweise bleibe der Künstler Eigentümer der Kunstwerke. Er habe als Künstler jedenfalls gem. § 950 BGB Eigentum an dem Kunstwerk erworben.

Es sei zwischen den Parteien ein Miet- oder Verwahrungsvertrag in Form eines gemischt-typischen Vertrages zustande gekommen und das Museum habe durch grobe Fahrlässigkeit bei der Lagerhaltung das Verschwinden der Fahnen zu vertreten. Es sei damals kein Werkvertrag zwischen dem Kläger als Künstler und der Landeshauptstadt München geschlossen worden, es habe kein Eigentumserwerb nach § 929 S. 1 BGB aufgrund einer Übereignung an die Beklagte stattgefunden und es läge auch keine Ersitzung der Fahnen vor. Maßgeblich führt der Kläger an, dass das Kunstwerk bei der Beklagten nicht inventarisiert worden sei, woraus zu schließen sei, dass die Beklagte keinen Eigentumswillen und Erwerbswillen gehabt habe. Zur Wiederherstellung der Fahnen sei auch ein Aufwand des Klägers als Künstler notwendig gewesen, den der Kläger mit 2.000,00 € ansetzt. Der materielle Schaden von 14.386,78 US-Dollar entspreche $11.500,00 €$.

Der Kläger beantragt daher, die Beklagte zu verurteilen, an den Kläger 13.500,00 € nebst Zinsen zu zahlen.
Die Beklagte beantragt, die Klage abzuweisen.

Sie trägt vor, die ursprüngliche Vereinbarung "Engagement" vom 14. Mai 1991 sei zur Vorbereitung auf die Ausstellung getroffen worden. Aus dieser Vereinbarung ergäbe sich, dass das Werkvertragsrecht gemäß §§ 631 ff. BGB Anwendung finden würde. Daraus ergäbe sich dann auch ein Eigentumsübergang zur Ausstellung des Werkes. Man habe schließlich 25.000,00 DM an den Künstler bezahlt. Eine Einlagerung des Werkes für den Künstler nach Abnahme der Fahnen sei mangels irgendwelcher Vereinbarungen nie erfolgt.

Die Beklagte erhebt jedenfalls die Einrede der Verjährung, treffend für eventuelle Ansprüche aus dem Mietrecht, aus dem Verwahrungsrecht und auch bezüglich der Schadensersatzansprüche. Des Weiteren führt die Beklagte das Rechtsinstitut der Verwirkung an, das auch in diesem Fall einschlägig sei. (Sachverhalt von der Redaktion bearbeitet.)

\section{Entscheidungsgründe}

I. Die zulässige Klage war überwiegend begründet. Der bestehende Schadensersatzanspruch aus Delikt bzw. Schlechterfüllung einer Gefälligkeitsverwahrung war wegen der Übergangsregelungen zur Verjährung im BGB noch nicht verjährt.

Anspruchsgrundlage für einen Schadensersatz wäre der $\S 280$ BGB, da die Herausgabe des Werkes heute jedenfalls unmöglich ist, in Verbindung mit einem Vertrag bzw. sogar einem Gefälligkeitsverhältnis oder einer GoA gem. §§ 677 ff. BGB.

Daneben wäre ein deliktischer Anspruch aus § 823 Abs. 1 BGB oder (§ 993 Abs. 1 aE BGB) ein Anspruch aus §§ 989, 990 BGB denkbar.

1. Einen vertraglichen Herausgabeanspruch - und damit über § 280 BGB einen Schadensersatzanspruch - vermag das Gericht nicht im Ansatz zu erkennen, die Argumentation des Klägers diesbezüglich scheint „an den Haaren“ herbeigezogen zu sein. Vielmehr liegt in diesem Fall die klassische Konstellation ohne Vorliegen einer verbindlichen Vereinbarung vor, die natürlich zu Missverständnissen führen muss. Die Parteien hatten nichts bezüglich der weiteren Aufbewahrung vereinbart. Wegen des erfolgten einstweiligen Ausstellungsverbotes lag ein ganz spezieller Einzelfall vor. Das Kunstwerk war (zumindest zu diesem Zeitpunkt) in Deutschland nicht mehr ausstellbar und damit war es für die Beklagte offenbar auch von untergeordnetem Interesse. Bei diesem Einzelfall, der sich in der laufenden Ausstellung ergeben hat, ist die Argumentation des Klägers, dass es Übung in Künstlerkreisen sei, Kunstwerke gegen ein Honorar für eine bestimmte Ausstellung anzufertigen, die dann nach Beendigung dieser Ausstellung für den Künstler durch das Museum bzw. den Veranstalter der Ausstellung (kurzfristig?) unentgeltlich aufbewahrt werden, fehl am Platz. Beweis braucht dazu folglich nicht erhoben zu werden. 
Zudem ist es in der heutigen pluralistischen Welt, in der Partikularinteressen vorherrschen, nicht vorstellbar, dass unentgeltlich relativ risikoreiche Verwahrungen vorgenommen werden. Die bloße Behauptung, dass es künstlerische Übung sei, kann nicht begründen, dass die Beklagte unentgeltlich für einen derart langen Zeitraum das Verwahrungsrisiko übernehmen wollte. Diese Überlegung ist letztlich schon bei Vertragsschluss anzustellen, so dass es wenig realistisch erscheint, dass die Beklagte in derartige Verträge eingewilligt hätte.

2. In solchen Fällen greifen die gesetzlichen Regelungen ein. Eine berechtigte GoA gem. § 677 BGB liegt wegen der vorläufig geduldeten Besitzberechtigung der Beklagten nicht vor. Damit bleibt ein Anspruch aus $\S 280$ BGB in Verbindung mit einem Gefälligkeitsverhältnis sowie der deliktische Anspruch aus $\S 823$ Abs. 1 BGB.

Die Regelungen der $\S$ 990, 993 Abs. 1 a. A. BGB sind bei der vorhandenen vorläufigen, nicht vindikationshindernden Besitzberechtigung nicht anwendbar; ganz h. M. Argument: systemwidrig und kein Bedürfnis der Anwendbarkeit neben § 823 BGB.

\section{a. Der Kläger ist Eigentümer des Kunstwerkes (geblieben).}

Mit Erstellung seines Kunstwerkes ist der Kläger natürlich zunächst über § 950 BGB Eigentümer geworden. Eine Übereignung an die Beklagte erfolgte nachfolgend nicht. Der Vertrag "Engagement" [...] betraf tatsächlich nur die Vorbereitung zum Ausstellungsprojekt, und das Honorar sollte die dem Künstler entstehenden Kosten abdecken, der Vortrag des Klägers dazu überzeugt, auch wegen der in dem Vertrag genannten Zeitpunkte und Abläufe. In den weiteren Telefonaten und Briefwechseln ging die Beklagte auch andeutungsweise noch von einem Eigentum des Künstlers aus, vgl. dazu die Anlagen des Klägers.

Vor allem aber inventarisierte die Beklagte das Kunstwerk nicht im Museumsbestand, was zeigt, dass sie offenbar (noch?) nicht Eigentümerin sein wollte. Damit scheidet auch eine Ersitzung durch die Beklagte gem. § 937 BGB nach zehn Jahren aus, da sie gerade keinen Eigenbesitz hatte. Dieser kann bei einem städtischen Museum aufgrund der öffentlich-rechtlichen Vorgaben nur angenommen werden, wenn das Kunstwerk inventarisiert ist.

b. Die rechtswidrige Pflichtverletzung ist bereits in der mangelnden Inventarisierung des Kunstwerkes im Jahre 1991 zu sehen.

Unstreitig wurde das Kunstwerk während der laufenden Veranstaltung im Jahre 1991 wegen des gerichtlichen Vorgehens der R. AG von der Beklagten abgenommen und gerade nicht inventarisiert. D.h. eine ordnungsgemäße Lagerhaltung wurde weder als Eigenbesitzer für die Beklagte selbst, noch als Fremdbesitzer für den Kläger durchgeführt. Das Kunstwerk war damit bereits dem Untergang geweiht, da der Verbleib nicht mehr zu rekonstruieren gewesen ist.
Dieses Nichtinventarisieren und damit Nicht-in-eine-ordnungsgemäße-Lagerung-Überführen, ist die einzig anknüpfbare Pflichtverletzung, die man der Beklagten vorwerfen kann. Der weitere Verbleib des Kunstwerkes ist genau deshalb auch heute nicht mehr zu rekonstruieren. Bei schon fehlender Inventarisierung stellt sich die Frage nach der Lagerhaltung des Museums im Übrigen nicht mehr, insbesondere nicht bei dem sonst ausgezeichneten Ruf, den das Lenbachhaus bei der Unterbringung seiner inventarisierten Kunstwerke genießt.

Diese Pflichtverletzung ist bereits im Jahre 1991 erfolgt. Die Tatsache, dass keine Inventarisierung erfolgte, ist von beiden Parteien so vorgetragen worden, Beweiserhebungen dazu bedarf es folglich nicht.

c. Diese Pflichtverletzung war auch grob fahrlässig, da ohne eine Notiz über den Verbleib das Kunstwerk der Gefahr des Untergangs/Verlustes ausgesetzt ist. Das muss jedem verantwortlich handelnden Museumsmitarbeiter sofort einleuchten.

d. Eine Verjährung der Schadensersatzansprüche lag nicht vor. Denn es gilt entweder die Dreijahresfrist §§ 195, 199 Abs. 1 BGB ab dem Jahre 2006 - Kenntnis vom Untergang des Kunstwerkes hatte der Kläger tatsächlich erst ab der neuerlichen Nachfrage im Mai 2006.

Oder unabhängig von der Kenntnis gem. § 199 Abs. 3 Satz 1 Nr. 1 und Satz 2 BGB in Verbindung mit Art. 229 EGBGB gilt die 10-jährige Höchstfrist, die aber erst am 01. Januar 2002 zu laufen begann. Es hätte nach alter Rechtslage gem. § 852 Abs. 1 a.E. BGB a.F. 30 Jahre Verjährungsfrist ab 1991 gegolten. (Anmerkung: Nach neuer Fassung des BGB wäre der Anspruch nach zehn Jahren ab 1991 verjährt!)

Neben diesen differenzierten Regelungen zur Verjährung verbietet sich die Anwendung des Billigkeitsinstrumentes der Verwirkung.

e. Die Schadenshöhe ist mit der Erstellung der Fahnen ausreichend dargelegt, ein eventueller Kursverlustschaden durch das zwischenzeitliche ungünstige Kursverhältnis zum US-Dollar ist ab Rechtshängigkeit als Verzugsschaden umfasst; 11.500,00 € können zugesprochen werden.

Der behauptete Arbeitsaufwand des Klägers von weiteren $2.000,00 €$ indes ist nicht im Geringsten substantiiert und offenbar nur unmotiviert der Klageforderung hinzugefügt worden [...]. Er ist weder belegt, noch nachvollziehbar und so der Beklagten nicht zumutbar. [...]

\section{II. [...] (Entscheidungsgründe von der Redaktion bearbeitet.)}

